\title{
Platelet isoform of phosphofructokinase promotes aerobic glycolysis and the progression of non-small cell lung cancer
}

\author{
FUAN WANG ${ }^{1},{\mathrm{LING} \mathrm{LI}^{2} \text { and } \mathrm{ZHEN} \mathrm{ZHANG}}^{3}$ \\ ${ }^{1}$ Department of Surgical Group, Medical College of Pingdingshan University, Pingdingshan, Henan 467000; \\ ${ }^{2}$ Department of Respiratory Medicine, First People's Hospital of Jinan, Jinan, Shandong 250000; \\ ${ }^{3}$ Department of Neurosurgery, Shandong Provincial Hospital, Jinan, Shandong 250012, P.R. China
}

Received April 2, 2020; Accepted October 19, 2020

DOI: $10.3892 / \mathrm{mmr} .2020 .11712$

\begin{abstract}
The platelet isoform of phosphofructokinase (PFKP) is a rate-limiting enzyme involved in glycolysis that serves an important role in various types of cancer. The aim of the present study was to explore the specific regulatory relationship between PFKP and non-small cell lung cancer (NSCLC) progression. PFKP expression in NSCLC tissues and corresponding adjacent tissues was detected using reverse transcription-quantitative polymerase chain reaction (RT-qPCR) and immunohistochemical analysis. PFKP expression in human bronchial epithelial cells (16HBE) and NSCLC cells (H1299, H23 and A549) was also detected using RT-qPCR. Cell proliferation was detected by Cell Counting Kit-8 and colony formation assays. Transwell invasion and wound healing assays, and flow cytometry were used to detect cell invasion, migration and apoptosis, respectively. The expression levels of glycolysis-associated enzymes (hexokinase-2, lactate dehydrogenase A and glucose transporter-1), epithelial-mesenchymal transition-related proteins (N-cadherin, vimentin and E-cadherin) and apoptosis-related proteins (caspase-3 and B-cell lymphoma-2) were detected
\end{abstract}

Correspondence to: Dr Zhen Zhang, Department of Neurosurgery, Shandong Provincial Hospital, 324 Jingwuwei 7th Road, Jinan, Shandong 250012, P.R. China

E-mail: zhangzhen7134@163.com

Abbreviations: ADP, adenosine diphosphate; ATP, adenosine triphosphate; BC, breast cancer; Bcl-2, B-cell lymphoma-2; CCK-8, Cell Counting Kit-8; EMT, epithelial-mesenchymal transition; FBS, fetal bovine serum; GBM, glioblastoma; Glut-1, glucose transporter-1; HCC, hepatocellular carcinoma; HK-2, hexokinase-2; HRP, horseradish peroxidase; IHC, immunohistochemistry; LDHA, lactate dehydrogenase A; NSCLC, non-small cell lung cancer; OD, optical density; PFK-1, phosphofructokinase-1; PFKL, liver isoform of phosphofructokinase; PFKP, platelet isoform of phosphofructokinase; PKM2, pyruvate kinase M2; RT-qPCR, reverse transcription-quantitative polymerase chain reaction; siRNA, small interfering RNA

Key words: PFKP, NSCLC, aerobic glycolysis, proliferation, EMT by western blotting. Glucose uptake, lactate production and the adenosine trisphosphate/adenosine diphosphate ratio were measured using the corresponding kits. The results of the present study demonstrated that PFKP expression was upregulated in NSCLC tissues and cells, and PFKP expression was related to lymph node metastasis and histological grade. In addition, overexpression of PFKP inhibited cell apoptosis, and promoted proliferation, migration, invasion and glycolysis of H1299 cells, whereas knockdown of PFKP had the opposite effects. In conclusion, PFKP inhibited cell apoptosis, and promoted proliferation, migration, invasion and glycolysis of NSCLC cells; these findings may lay the foundation for novel treatments of NSCLC.

\section{Introduction}

Lung cancer is one of the major diseases endangering human health worldwide (1). The incidence and mortality rates of lung cancer are high, particularly non-small cell lung cancer (NSCLC) $(2,3)$. Invasion and metastasis are the main causes of poor prognosis and high mortality rate in patients with lung cancer (4). Although great efforts, such as the identification of novel biomarkers, establishment of modified operations and development of specific drugs, have been made in the clinical setting, the postoperative survival rate of patients has not significantly improved $(5,6)$. Therefore, it is of great clinical significance to explore the molecular mechanisms underlying the progression of NSCLC.

Glucose metabolism is one of the most distinguishing characteristics between normal and tumorigenic cells (7). Tumor cells mainly rely on glycolysis to obtain energy, even in aerobic environments; this is known as the Warburg effect (8). A previous study revealed that the growth and survival of tumor cells depend on the transformation from anaerobic glycolysis to aerobic glycolysis (9). Aerobic glycolysis provides a material basis for the survival, growth and proliferation of tumor cells (10). Furthermore, a large amount of lactic acid causes damage to the body, which is conducive to tumor tissues invading adjacent normal tissues (11). In recent years, the Warburg effect has garnered much attention in the study of tumor development mechanisms $(12,13)$. Although the Warburg effect has been recognized in NSCLC (14-16), the driving mechanism of aerobic glycolysis remains unknown. 
The platelet isoform of phosphofructokinase (PFKP) is a rate-limiting enzyme involved in glycolysis, which can cause the conversion of fructose 6-phosphate to fructose 1,6-bisphosphate (17). Enhanced activity of PFKP can increase glycolysis, increase fatty acid oxidation, and promote DNA biosynthesis and repair, thereby enhancing tumor cell growth, migration and invasion $(18,19)$. It has been reported that PFKP expression is upregulated in various types of cancer, including breast cancer (BC) (20), hepatocellular carcinoma (HCC) (17) and renal carcinoma (21). However, to the best of our knowledge, the specific regulatory relationship between PFKP and NSCLC progression remains unclear.

The present study aimed to assess the effects of PFKP on NSCLC progression, and further investigated the effects of PFKP on cell proliferation, apoptosis, migration, invasion and glycolysis. The present findings may provide a basis for novel strategies for the treatment of NSCLC.

\section{Materials and methods}

The Cancer Genome Atlas (TCGA) data analysis. The mRNA gene expression data were downloaded from TCGA LUAD datasets (https://portal.gdc.cancer.gov/). Overall survival information of all TCGA datasets was obtained from supplementary data (22). For differentially expressed gene (DEG) analysis, PFKP mRNA expression in cancer tissues and paracancerous (normal) tissues was analyzed using the DESeq2 R package (23); the adjusted P-value for each gene was calculated using the false-discovery rate (FDR) method. The cut-off to detect DEGs was an FDR of $<0.05$ and an absolute $\log 2$-fold change of $>1$. For overall survival analysis, data analysis was performed in $\mathrm{R}$ package 'survival' (24) using the Kaplan-Meier curve method, and a log-rank test was used to compare survival times between two groups.

Patient and tissue samples. In the present study, NSCLC tissues and corresponding adjacent tissues ( $>2 \mathrm{~cm}$ away from the tumor edge) were collected from 84 patients who underwent primary surgical resection of NSCLC between January 2018 and December 2019 at Shandong Provincial Hospital (Jinan, China). None of the patients received radiotherapy or chemotherapy prior to surgical resection, and the diagnosis of NSCLC was assessed by an experienced pathologist. All tissue samples were stored at $-80^{\circ} \mathrm{C}$ until RNA extraction. The present study was approved by the Research Ethics Committee of Shandong Provincial Hospital (approval no. 2020-181; Jinan, China), and each patient provided written informed consent.

Immunohistochemistry (IHC). All tissue samples were fixed with $4 \%$ formaldehyde solution for $24 \mathrm{~h}$ at room temperature and embedded in paraffin. Paraffin-embedded tissues were cut into 4- $\mu \mathrm{m}$ sections, which were routinely dehydrated, boiled in $0.01 \mathrm{M}$ citrate buffer ( $\mathrm{pH}$ 6.1) for $2 \mathrm{~min}$ for antigen retrieval and incubated with $3 \% \mathrm{H}_{2} \mathrm{O}_{2}$ at room temperature for $15 \mathrm{~min}$. The slides were then incubated with primary antibodies against PFKP (1:150; cat. no. ab119796), hexokinase-2 (HK-2; 1:500; cat. no. ab209847) and pyruvate kinase M2 (PKM2; 1:500; cat. no. ab137852) (all Abcam) at $4^{\circ} \mathrm{C}$ overnight. Subsequently, the slides were incubated with a secondary antibody labeled with horseradish peroxidase (HRP) (1:2,000; cat. nos. ab205719 or ab205718; Abcam) for $30 \mathrm{~min}$ at room temperature and 3'-diaminobenzidine reagent (Beijing Solarbio Science \& Technology Co., Ltd.) was added to the slides for visualization. Finally, the sections were dehydrated, cleared with xylene and fixed with resin. Two experienced pathologists independently analyzed the IHC results. Brown particles were considered positive expression of PFKP, HK-2 and PKM2.

Cell culture. Human bronchial epithelial cells (16HBE) and NSCLC cells (H1299, H23 and A549) were purchased from American Type Culture Collection. All cells were grown in RPMI-1640 medium (Gibco; Thermo Fisher Scientific, Inc.) supplemented with $10 \%$ fetal bovine serum (FBS; Gibco; Thermo Fisher Scientific, Inc.) and cultured in an incubator containing $5 \% \mathrm{CO}_{2}$ at $37^{\circ} \mathrm{C}$.

Cell transfection. $\mathrm{H} 1299$ cells were divided into control (without treatment), pVMV6-ENTRY [negative control (NC); cells were transfected with pCMV6 empty plasmid] and pVMV6-PFKP (overexpression of PFKP; cells were transfected with pCMV6-PFKP plasmid) groups. H23 cells were divided into control (without treatment), small interfering RNA (siRNA/si)-NC (NC; cells were transfected with NC siRNAs), si1-PFKP (knockdown of PFKP; cells were transfected with si1-PFKP) and si2-PFKP (knockdown of PFKP; cells were transfected with si2-PFKP) groups. The plasmids (50 ng) and siRNAs $(50 \mathrm{nM})$ were transfected into cells $\left(1 \times 10^{4}\right.$ cells/well $)$ using Lipofectamine ${ }^{\circledR} 3000$ (Invitrogen; Thermo Fisher Scientific, Inc.) according to the manufacturer's instructions. The pCMV6-PFKP, pVMV6-ENTRY, siRNA and siRNA-NC were purchased from OriGene Technologies, Inc. The sequences for the siRNAs were as follows: si-NC sense, 5'-GGC UACGUCCAGGAGCGCAUU-3' and antisense, 5'-UGCGCU CCUGGACGUAGCCUU-3'; si1-PFKP sense, 5'-GCUCCA UUCUUGGGACAAATT-3' and antisense, 5'-AGAUUCUGG AAGGAACACCTT-3'; si2-PFKP sense, 5'-GGUGUUCCU UCCAGAAUCUTT-3' and antisense, 5'-UUUGUCCCAAGA AUGGAGCTT-3'; and PFKP sense, 5'-GGACGCGGACGA CTCCCGGGC-3' and antisense, 5'-GTCAGACACTCCAGG GCTGCACATGTTCC-3'. A total of $48 \mathrm{~h}$ post-transfection, follow-up experiments were conducted.

Reverse transcription-quantitative PCR (RT-qPCR). Total RNA was extracted from tissues and cells using TRIzol ${ }^{\circledR}$ reagent (Invitrogen; Thermo Fisher Scientific, Inc.) according to the manufacturer's instructions.cDNA was synthesized using the PrimeScript RT reagent kit (Invitrogen; Thermo Fisher Scientific, Inc.) according to the manufacturer's protocol. RT-qPCR was performed using the SYBR Green PCR kit (Takara Biotechnology Co., Ltd.) and was monitored using the CFX96 Touch Real-Time PCR Detection system (Bio-Rad Laboratories, Inc.). The reaction conditions were as follows: Pre-denaturation at $95^{\circ} \mathrm{C}$ for $3 \mathrm{~min}$, followed by 40 cycles of denaturation at $95^{\circ} \mathrm{C}$ for $15 \mathrm{sec}$, annealing at $58^{\circ} \mathrm{C}$ for $45 \mathrm{sec}$ and extension at $72^{\circ} \mathrm{C}$ for $31 \mathrm{sec}$. Relative mRNA expression was analyzed using the $2^{-\Delta \Delta C q}$ method (25). GAPDH was used as an endogenous control. The primers (Table I) were synthesized by Invitrogen; Thermo Fisher Scientific, Inc. 
Table I. Primer sequences.

\begin{tabular}{ll}
\hline Primers & \multicolumn{1}{c}{ Sequences $\left(5^{\prime}-3^{\prime}\right)$} \\
\hline PFKP-F & GGAGTGGAGTGGGCTGCTGGAG \\
PFKP-R & CATGTCGGTGCCGCAGAAATCA \\
GAPDH -F & TGTTG CCATCAATGACCCCTT \\
GAPDH -R & CTCCACGACGTACTCAGCG
\end{tabular}

PFKP, platelet isoform of phosphofructokinase; F, forward; R, reverse.

Western blot analysis. Radioimmunoprecipitation assay buffer (Thermo Fisher Scientific, Inc.) was used to extract total proteins from NSCLC cells. A bicinchoninic acid protein assay kit (Beyotime Institute of Biotechnology) was used to calculate protein concentration. Total proteins $(30 \mu \mathrm{g})$ were separated by SDS-PAGE on $10 \%$ gels and were transferred to polyvinylidene fluoride membranes. After blocking with 5\% fat-free milk in TBS-0.1\% Tween-20 for $1 \mathrm{~h}$ at room temperature, the membranes were incubated with the following primary antibodies at $4^{\circ} \mathrm{C}$ overnight: PFKP (cat. no. 12746), N-cadherin (cat. no. 13116), E-cadherin (cat. no. 3195), vimentin (cat. no. 5741), caspase-3 (cat. no. 9662) B-cell lymphoma-2 (Bcl-2; cat. no. 4223), hexokinase-2 (HK-2; cat. no. 2867), lactate dehydrogenase A (LDHA; cat. no. 3582), GAPDH (cat. no. 5174) (all 1:1,000; all from Cell Signaling Technology, Inc.) and glucose transporter-1 (Glut-1; cat. no. ab115730; 1:1,000; Abcam). GAPDH was used as the internal control. Subsequently, the membranes were incubated with HRP-conjugated goat anti-rabbit antibody (cat. no. A0208; 1:4,000; Beyotime Institute of Biotechnology) for $1 \mathrm{~h}$ at room temperature. Finally, an ECL western blotting substrate (cat. no. 32106; Pierce; Thermo Fisher Scientific, Inc.) was used to visualize protein bands and the specific bands were semi-quantified using ImageJ v1.8.0 (National Institutes of Health).

Cell proliferation and colony formation assays. Cell proliferation was measured by performing the Cell Counting Kit-8 (CCK-8) assay (Beyotime Institute of Biotechnology). Briefly, cells $\left(2 \times 10^{3}\right.$ cells) were seeded on 96 -well plates. After seeding for $0,24,48,72$ and $96 \mathrm{~h}$, the cells were incubated with CCK-8 solution $(10 \mu \mathrm{l} / \mathrm{ml})$ at $37^{\circ} \mathrm{C}$ for $2 \mathrm{~h}$ in the dark. The optical density (OD) was measured at $450 \mathrm{~nm}$ using a spectrophotometer (Thermo Fisher Scientific, Inc.).

For the colony formation assay, cells (500 cells) were seeded on 6-well plates and maintained in RPMI-1640 medium containing $10 \%$ FBS for 14 days. The medium was replaced every 3 days. Before acquiring the images under the light microscope (Olympus Corporation; magnification, x5), the cells were fixed with $4 \%$ formaldehyde for $15 \mathrm{~min}$ at room temperature and stained with $0.1 \%$ crystal violet for $20 \mathrm{~min}$ at room temperature. More than 50 cells in a colony were recorded as effective clones.

Wound healing assay. Cells were seeded on 6-well plates and cultured to $90-100 \%$ confluence. A $200-\mu$ l pipette tip was used to create wounds across the cell monolayer. After incubation in a serum-free RPMI-1640 medium for $48 \mathrm{~h}$, the migration distance was observed. The wound healing rate was calculated according to the following formula: Wound healing rate $(\%)=($ wound area at $0 \mathrm{~h}$-wound area at $24 \mathrm{~h}) /$ wound area at $0 \mathrm{~h} \times 100$.

Transwell invasion assay. Transwell chambers (pore size, $8 \mu \mathrm{m}$; Corning, Inc.) were used to investigate the invasive ability of the cells. Briefly, a total of $200 \mu \mathrm{l}$ cell suspension containing $1 \times 10^{5}$ cells was added to the upper chamber, which was coated with Matrigel, and $600 \mu 1$ medium containing $10 \%$ FBS was added to the lower chamber. After incubation for $48 \mathrm{~h}$ in a $\mathrm{CO}_{2}$ incubator at $37^{\circ} \mathrm{C}$, cotton swabs were used to remove cells from the upper surface. Subsequently, the cells were fixed with $2 \%$ paraformaldehyde for $10 \mathrm{~min}$ at room temperature and stained with $0.1 \%$ crystal violet for $10 \mathrm{~min}$ at room temperature. Finally, the number of invasive cells was calculated in five random fields under an inverted light microscope (Olympus Corporation; magnification, x100).

Flow cytometric analysis of apoptosis. Cell apoptosis was detected by performing flow cytometry using an Annexin V-FITC/PI apoptosis kit (BD Biosciences). Briefly, cells were resuspended in binding buffer $(500 \mu \mathrm{l})$ and adjusted to $1 \times 10^{6}$ cells $/ \mathrm{ml}$, and Annexin V $(5 \mu \mathrm{l})$ and PI $(5 \mu \mathrm{l})$ were added to the buffer. After incubation for $15 \mathrm{~min}$ at room temperature in the dark, the apoptotic rate was analyzed by flow cytometry (Accuri C6 Plus; BD Biosciences).

Detection of glucose uptake, lactate production and the adenosine triphosphate (ATP)/adenosine diphosphate (ADP) ratio. The glucose test kit (cat. no. K686-100), lactate assay kit (cat. no. K627-100) and ApoSENSOR ${ }^{\mathrm{TM}}$ ADP/ATP ratio bioluminescence assay kit (cat. no. K255-200) were purchased from BioVision, Inc. For the glucose uptake and lactate production assays, cells $\left(2.5 \times 10^{5}\right.$ cells) were seeded in 6 -well plates. After culturing for 0 and $48 \mathrm{~h}$, glucose uptake and lactate production were measured according to the manufacturer's instructions. Glucose uptake was referred to as the difference between the initial (0 h) and final (48 h) glucose levels, and lactate production was referred to as the difference between the final (48 h) and initial $(0 \mathrm{~h})$ lactate levels. To detect the ATP/ADP ratio, cells $\left(1 \times 10^{4}\right.$ cells) were seeded on a luminometer plate and incubated with nucleotide-releasing buffer. Subsequently, the ATP Monitoring Enzyme ( $1 \mu \mathrm{l})$ was added to the luminometer plate. After incubation for 1 and $10 \mathrm{~min}$, sample values were recorded (the value recorded at $1 \mathrm{~min}$ was recorded as $\mathrm{A}$, whereas the value recorded at $10 \mathrm{~min}$ was recorded as $\mathrm{B}$ ). Finally, ADP-converting enzyme $(1 \mu \mathrm{l})$ was added, and the sample values were recorded (the value was recorded as $\mathrm{C}$ ) after incubation for $2 \mathrm{~min}$ at room temperature. The ATP/ADP ratio was calculated according to the following formula: $\mathrm{ATP} / \mathrm{ADP}$ ratio $=\mathrm{A} /(\mathrm{C}-\mathrm{B})$.

Statistical analysis. Data are presented as the mean \pm standard deviation. GraphPad Prism 7.0 (GraphPad Software, Inc.) and SPSS 15.0 statistical software (SPSS, Inc.) were used for the statistical analyses. The $\chi^{2}$ test was used to analyze the association between PFKP expression and the clinicopathological parameters of patients. The paired Student's t-test was used for comparisons between the two groups. One-way analysis 
A

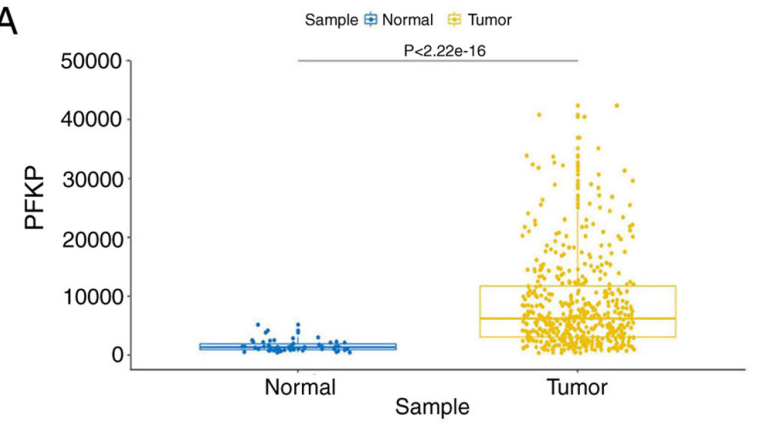

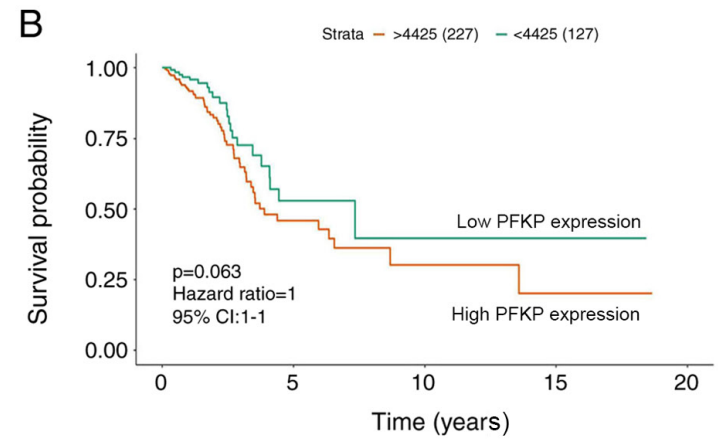

C

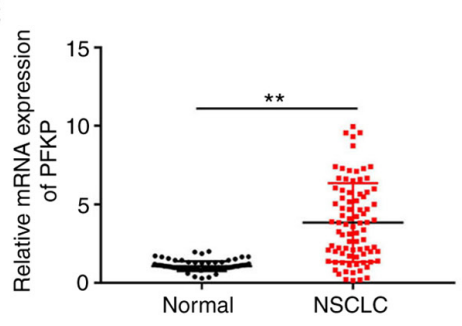

E

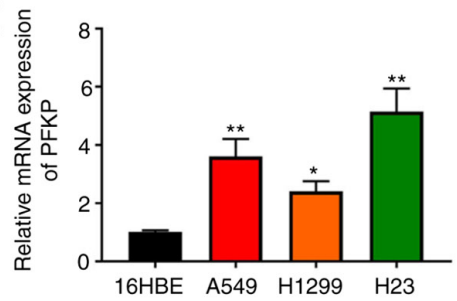

D
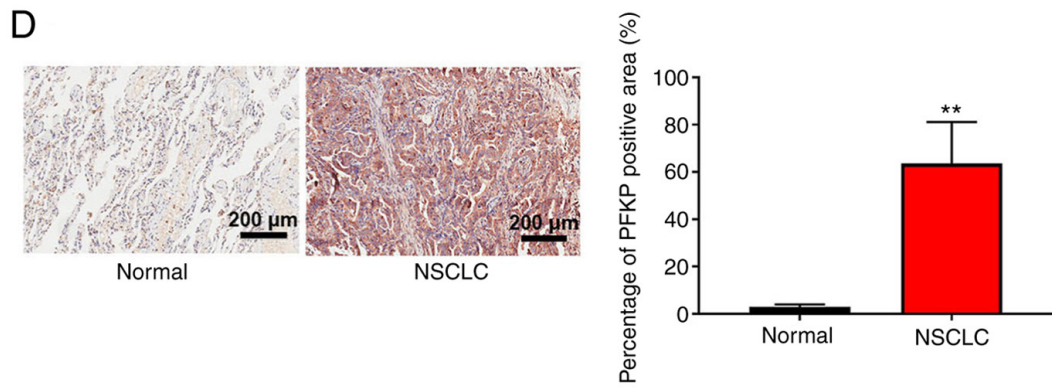

$\mathrm{F}$

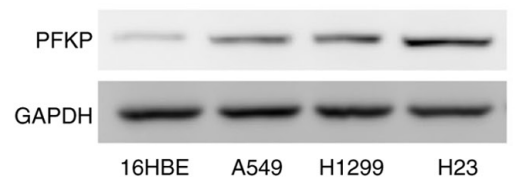

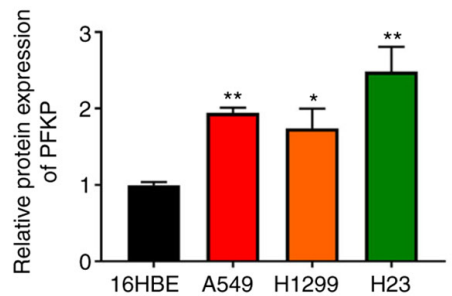

Figure 1. Upregulation of PFKP expression in NSCLC tissue and cells. (A) PFKP expression in NSCLC tissues and normal tissues was analyzed using data from TCGA. (B) Survival analysis based on TCGA data. (C) RT-qPCR was used to detect the mRNA expression levels of PFKP in NSCLC and corresponding adjacent tissues (normal) (n=84). (D) Immunohistochemical analysis was used to detect PFKP expression in NSCLC and corresponding adjacent tissues (normal) $(\mathrm{n}=84)$; scale bar, $200 \mu \mathrm{m}$. ${ }^{* *} \mathrm{P}<0.01 \mathrm{vs}$. normal tissues. (E) RT-qPCR was used to detect the mRNA expression levels of PFKP in 16HBE cells and NSCLC cells (H1299, H23 and A549). (F) Western blotting was used to detect the protein expression levels of PFKP in 16HBE cells and NSCLC cells (H1299, $\mathrm{H} 23$ and A549). Data are presented as the mean \pm SD from three independent experiments. ${ }^{*} \mathrm{P}<0.05,{ }^{* *} \mathrm{P}<0.01$ vs. $16 \mathrm{HBE}$ cells. PFKP, platelet isoform of phosphofructokinase; NSCLC, non-small cell lung cancer; TCGA, The Cancer Genome Atlas; RT-qPCR, reverse transcription-quantitative PCR.

of variance with Tukey's post-hoc test was used to compare multiple groups. $\mathrm{P}<0.05$ was considered to indicate a statistically significant difference.

\section{Results}

Upregulation of PFKP expression in NSCLC tissue and cells, and its association with clinicopathological parameters. According to TCGA data, PFKP expression was higher in NSCLC tissues compared with that in normal tissues $\left(\mathrm{P}=2.22 \times 10^{-16}\right.$; Fig. $\left.1 \mathrm{~A}\right)$. In addition, although overall survival did not exhibit a significant difference, Kaplan-Meier survival analysis revealed that the overall survival in patients with NSCLC and high PFKP expression was shorter than that in patients with NSCLC and low PFKP expression $(\mathrm{P}=0.063$; Fig. $1 \mathrm{~B})$. These data suggested that PFKP may be associated with poor prognosis in NSCLC. The present study further examined PFKP expression in NSCLC and corresponding adjacent tissues (normal) using RT-qPCR and IHC. As shown in Fig. 1C and D, PFKP expression in NSCLC tissues was upregulated compared with that in normal tissues $(\mathrm{P}<0.01)$. According to the median mRNA expression of PFKP, patients with NSCLC were divided into high expression and low expression groups. Subsequently, the association between PFKP expression and patient clinicopathological parameters was assessed. As shown in Table II, PFKP expression was associated with lymph node metastasis and histological grade (both $\mathrm{P}<0.01$ ), but there was no significant association between PFKP expression and other clinical characteristics $(\mathrm{P}>0.05)$. Furthermore, PFKP expression was detected in 16HBE cells and NSCLC cells (H1299, H23 and A549) using RT-qPCR and western blotting. As shown in Fig. $1 \mathrm{E}$ and F, compared with those in 16HBE cells, PFKP expression levels were upregulated in NSCLC cells $(\mathrm{P}<0.05)$. Moreover, $\mathrm{H} 23$ cells expressed relatively high levels of PFKP, whereas H1299 cells expressed relatively low levels of PFKP; therefore, H1299 and H23 cells were used in subsequent experiments.

PFKP promotes the proliferation of NSCLC cells. To examine the effects of PFKP on NSCLC cells, H1299 cells were transfected with pCMV6-PFKP plasmid, and H23 cells were transfected with siRNA-PFKP. The results of RT-qPCR (Fig, 2A) revealed that the mRNA expression levels of PFKP 
Table II. Association of PFKP expression with the clinicopathological parameters of patients.

\section{PFKP expression}

Clinicopathological parameters

No. of cases

High

Low

P-value

Sex

Male

Female

Age (years)

$<60$

$\geq 60$

Tumor size (cm)

$<3$

$\geq 3$

Lymph node metastasis

No
Yes

Histological grade

I

II-III
50

34

44

40

34

50

40

44

38

46
28

14

24

18

15

27

$0.009^{\mathrm{a}}$

Data were assessed by $\chi^{2}$ test. ${ }^{\mathrm{a}} \mathrm{P}<0.01,{ }^{\mathrm{b}} \mathrm{P}<0.001 \mathrm{vs}$. high PFKP expression group. PFKP, platelet isoform of phosphofructokinase.

A

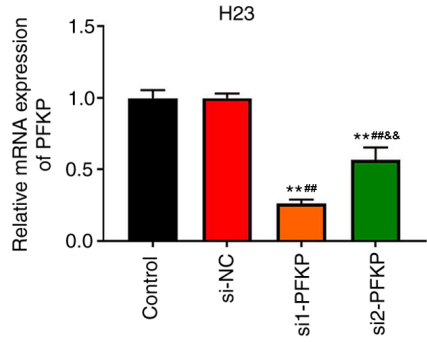

B

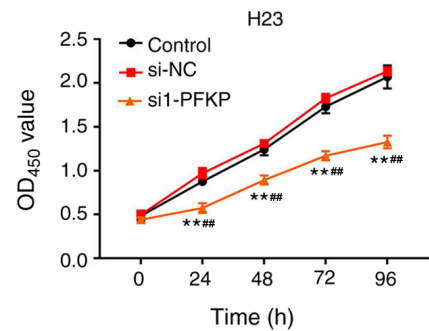

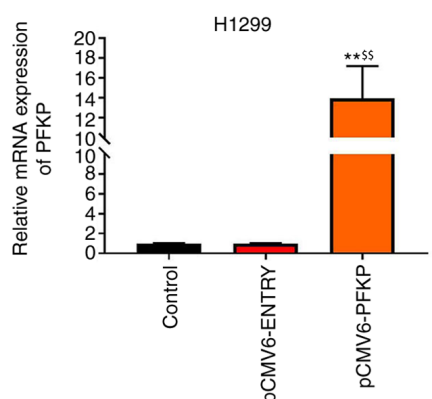

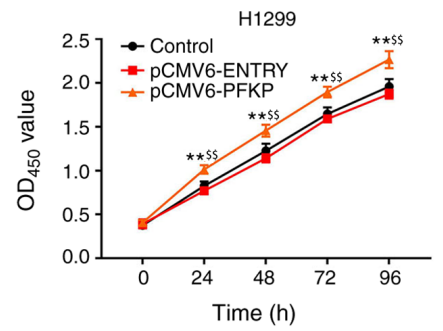

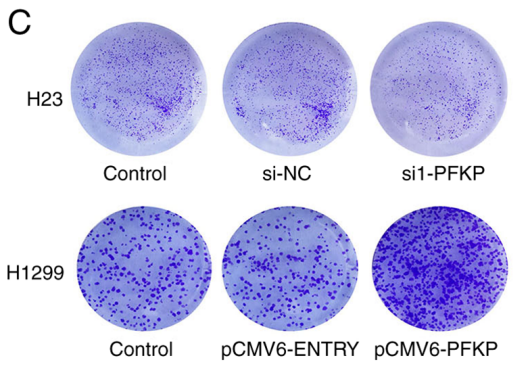
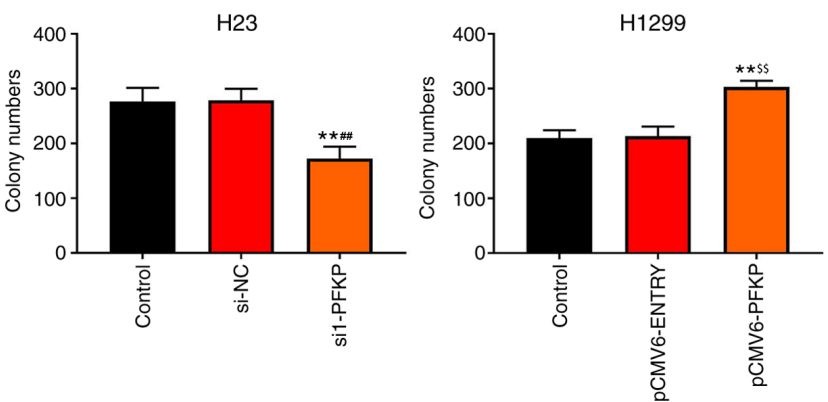

Figure 2. PFKP promotes the proliferation of non-small cell lung cancer cells. (A) mRNA expression levels of PFKP in H1299 and H23 cells were determined by reverse transcription-quantitative PCR. (B) Viability of H1299 and H23 cells was detected by Cell Counting Kit-8 assay. (C) Colony formation assay was used to detect the proliferation of $\mathrm{H} 1299$ and $\mathrm{H} 23$ cells. Data are presented as the mean \pm SD from three independent experiments. "* $\mathrm{P}<0.01$ vs. control group; ${ }^{\# \#} \mathrm{P}<0.01$ vs. si-NC group; \&\& $\mathrm{P}<0.01$ vs. sil-PFKP group; ${ }^{\$ \$} \mathrm{P}<0.01$ vs. pCMV6-ENTRY group. PFKP, platelet isoform of phosphofructokinase; OD, optical density; si, small interfering RNA; NC, negative control. 
A

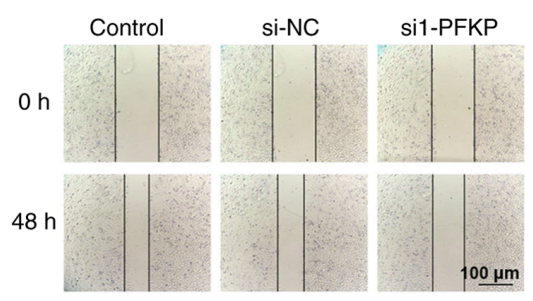

$\mathrm{H} 23$

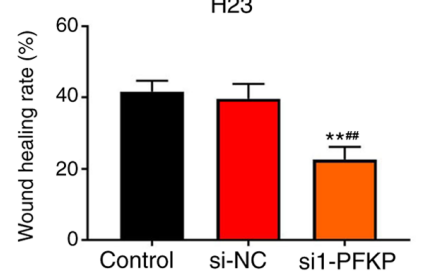

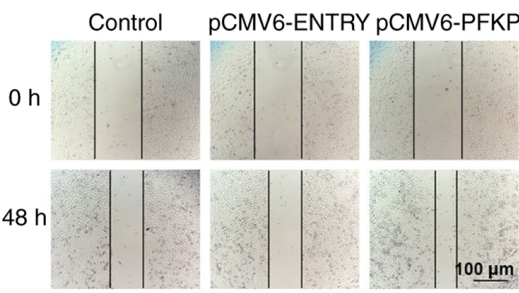

H1299

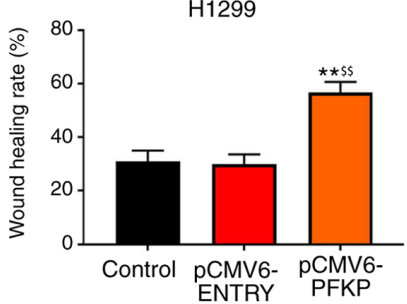

B
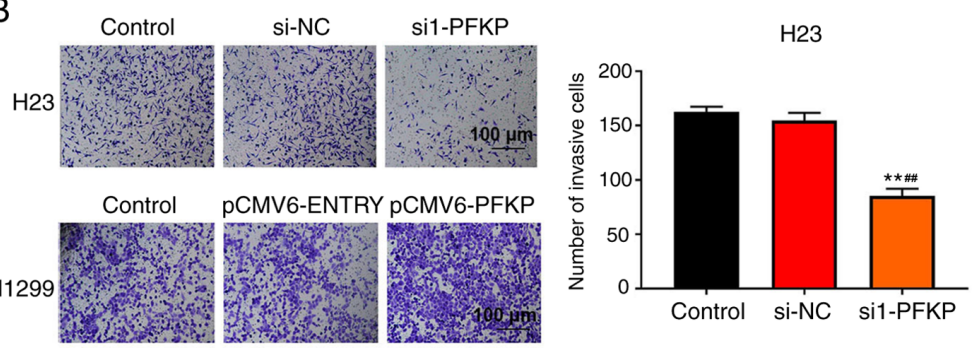

$\mathrm{H} 1299$

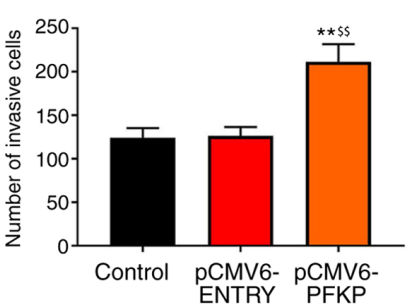

C
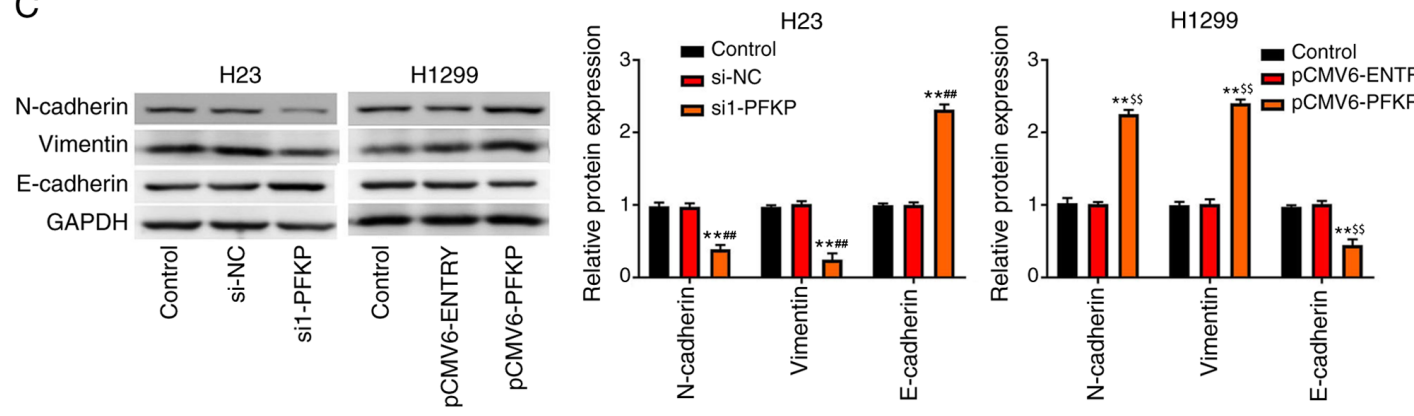

Figure 3. PFKP promotes migration, invasion and EMT of non-small cell lung cancer cells. (A) Wound healing assay was used to detect the migration of H23 and H1299 cells. (B) Transwell assay was used to detect the invasion of H23 and H1299 cells. (C) Expression levels of EMT-related proteins (N-cadherin, vimentin and E-cadherin) in H1299 and H23 cells were determined by western blot analysis. Data are presented as the mean \pm SD from three independent experiments. ${ }^{* *} \mathrm{P}<0.01$ vs. control group; ${ }^{\# \#} \mathrm{P}<0.01$ vs. si-NC group; ${ }^{\$} \mathrm{P}<0.01$ vs. pCMV6-ENTRY group. PFKP, platelet isoform of phosphofructokinase; EMT, epithelial-mesenchymal transition; si, small interfering RNA; NC, negative control.

were downregulated in the si1-PFKP and si2-PFKP groups $(\mathrm{P}<0.01)$, whereas the mRNA expression levels of PFKP were upregulated in the pCMV6-PFKP group $(\mathrm{P}<0.01)$, compared with in the control and si-NC or pCMV6-ENTRY groups. The results indicated that transfection was successful. Moreover, the mRNA expression levels of PFKP in the si1-PFKP group were significantly decreased compared with the si2-PFKP group $(\mathrm{P}<0.01$; Fig. $2 \mathrm{~A})$; therefore, sil-PFKP was used in subsequent experiments. The effects of PFKP on the proliferation of NSCLC cells were assessed using CCK-8 and colony formation assays. As shown in Fig. 2B, the OD450 value in the si1-PFKP group was lower than that in the control and si-NC groups $(\mathrm{P}<0.01)$; however, the OD450 value in the pCMV6-PFKP group was higher than that in the control and pCMV6-ENTRY groups $(\mathrm{P}<0.01)$. Moreover, similar results were obtained from the colony formation assay $(\mathrm{P}<0.01$; Fig. 2C).
PFKP promotes migration, invasion and epithelial -mesenchymal transition (EMT) of NSCLC cells. Transwell invasion and wound healing assays were used to detect the invasion and migration of NSCLC cells. As shown in Fig. 3A and B, overexpression of PFKP increased the wound healing rate and number of invasive cells among H1299 cells compared with those in the control and pCMV6-ENTRY groups $(\mathrm{P}<0.01)$, whereas knockdown of PFKP decreased the wound healing rate and number of invasive cells among $\mathrm{H} 23$ cells compared with those in the control and si-NC groups $(\mathrm{P}<0.01)$. In addition, western blot analysis (Fig. 3C) demonstrated that the protein expression levels of $\mathrm{N}$-cadherin and vimentin were significantly lower in the sil-PFKP group than those in the control and si-NC groups $(\mathrm{P}<0.01)$, whereas the protein expression levels of E-cadherin were increased $(\mathrm{P}<0.01)$. When PFKP expression was upregulated, the protein expression levels of $\mathrm{N}$-cadherin and vimentin were increased compared with those 

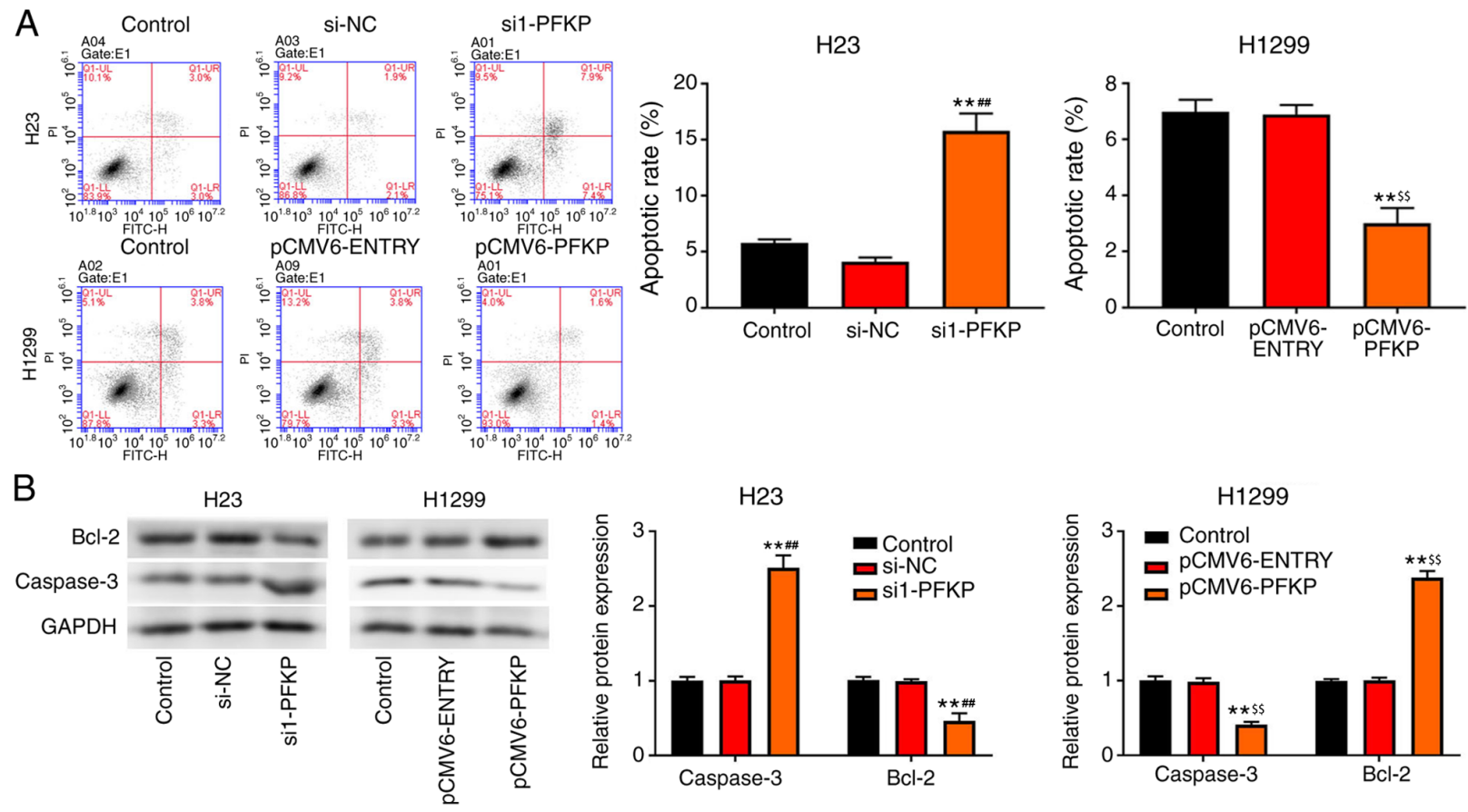

Figure 4. PFKP inhibits apoptosis of non-small cell lung cancer cells. (A) Apoptosis of H23 and H1299 cells was detected by flow cytometry. (B) Expression levels of apoptosis-related proteins (caspase-3 and Bcl-2) were detected by western blot analysis. Data are presented as mean \pm SD from three independent experiments. ${ }^{* *} \mathrm{P}<0.01$ vs. control group; ${ }^{* \#} \mathrm{P}<0.01$ vs. si-NC group; ${ }^{\$ \$} \mathrm{P}<0.01$ vs. pCMV6-ENTRY group. PFKP, platelet isoform of phosphofructokinase; Bcl-2, B-cell lymphoma 2; si, small interfering RNA; NC, negative control.

in the control and pCMV6-ENTRY groups $(\mathrm{P}<0.01)$, whereas the protein expression levels of E-cadherin were decreased $(\mathrm{P}<0.01)$.

PFKP inhibits apoptosis of NSCLC cells. Apoptosis of $\mathrm{H} 23$ and H1299 cells was detected using flow cytometry. As shown in Fig. 4A, overexpression of PFKP decreased the apoptotic rate in H1299 cells compared with that in the control and pCMV6-ENTRY groups $(\mathrm{P}<0.01)$, whereas an opposite trend in apoptotic rate was observed in the si1-PFKP group of H23 cells. Moreover, the expression levels of apoptosis-related proteins (caspase-3 and Bcl-2) were detected using western blot analysis. As presented in Fig. 4B, overexpression of PFKP reduced the protein expression levels of caspase- 3 and increased the protein expression levels of Bcl-2 in H1299 cells compared with those in the control and pCMV6-ENTRY groups $(\mathrm{P}<0.01)$, whereas the opposite results were observed in the si1-PFKP group of $\mathrm{H} 23$ cells.

PFKP promotes glycolysis of NSCLC cells. The present study also investigated the effect of PFKP on glucose metabolism. The results revealed that overexpression of PFKP enhanced glucose uptake, lactate production and the ATP/ADP ratio in H1299 cells compared with those in the control and pCMV6-ENTRY groups $(\mathrm{P}<0.01)$, whereas knockdown of PFKP reduced glucose uptake, lactate production and the ATP/ADP ratio in $\mathrm{H} 23$ cells compared with those in the control and si-NC groups $(\mathrm{P}<0.01$; Fig. 5A-C). Moreover, the expression levels of glycolysis-associated enzymes (HK-2, LDHA and Glut-1) were detected using western blot analysis. As shown in Fig. 5D, overexpression of PFKP increased the protein expression levels of HK-2, LDHA and Glut-1 in H1299 cells compared with those in the control and pCMV6-ENTRY groups $(\mathrm{P}<0.01)$, whereas knockdown of PFKP decreased the protein expression levels of HK-2, LDHA and Glut-1 in $\mathrm{H} 23$ cells compared with those in the control and si-NC groups $(\mathrm{P}<0.01)$.

PFKP may increase the expression of HK-2 and PKM2 and regulate glycolysis in NSCLC specimens. To further determine the clinical significance of PFKP in glycolysis, the expression of glycolysis-associated enzymes (HK-2 and PKM2) was detected in patient samples (NSCLC and corresponding adjacent tissues) using IHC. As shown in Fig. 6, the expression levels of HK-2 and PKM2 were significantly higher in NSCLC tissues than those in normal tissues $(\mathrm{P}<0.01)$. These results suggested that PFKP may increase the expression of HK-2 and PKM2, and regulate glycolysis in NSCLC.

\section{Discussion}

NSCLC presents as fatal malignant tumors, and $\geq 65 \%$ of patients with NSCLC exhibit cancer progression with local advanced or metastatic features (26). PFKP is a second rate-limiting enzyme that has an important role in glycolysis and affects tumor progression (27). In the present study, PFKP expression was revealed to be upregulated in NSCLC tissues and cells, and was associated with lymph node metastasis and histological grade. In addition, the present findings demonstrated that overexpression of PFKP inhibited cell apoptosis, and promoted proliferation, migration, invasion, EMT and glycolysis in NSCLC cells, whereas knockdown of PFKP had the opposite effect. 

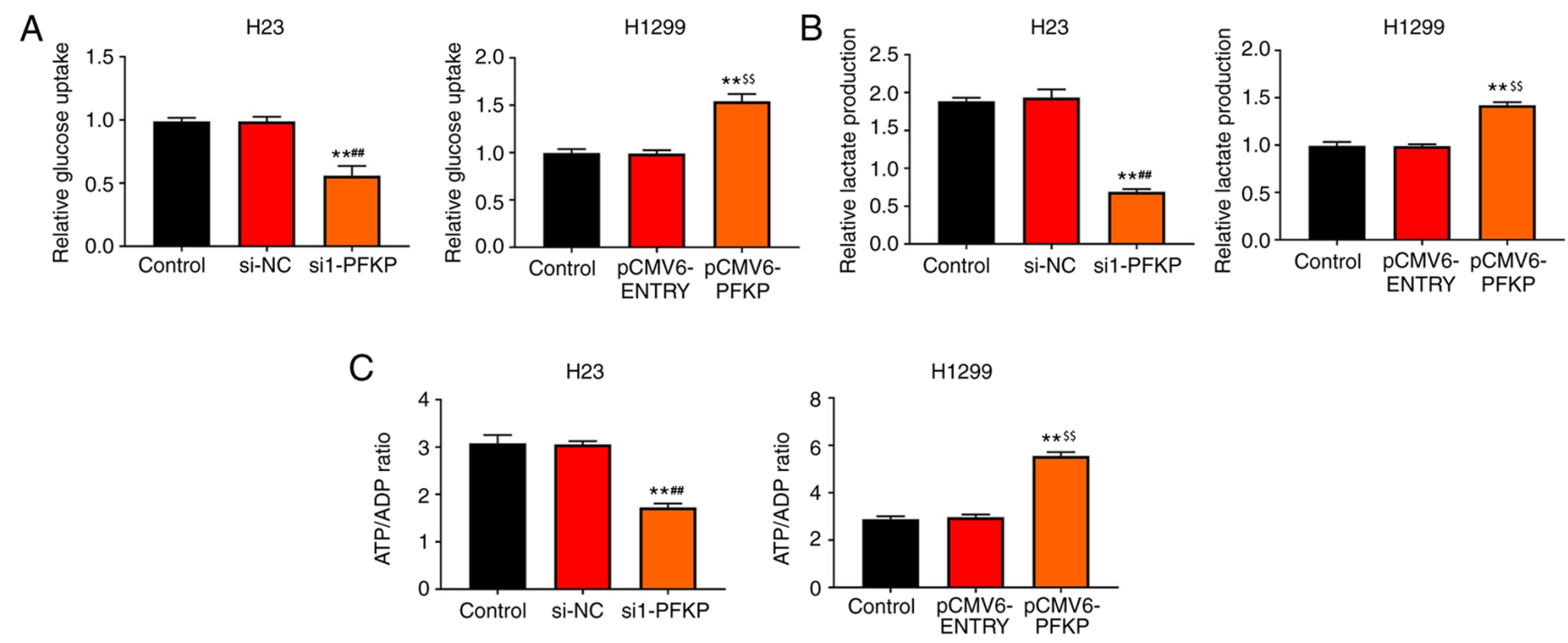

D

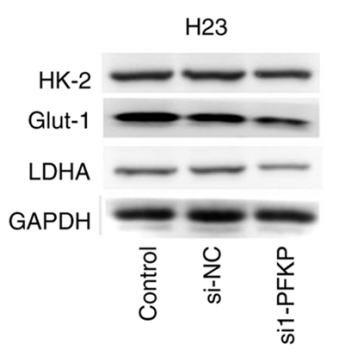

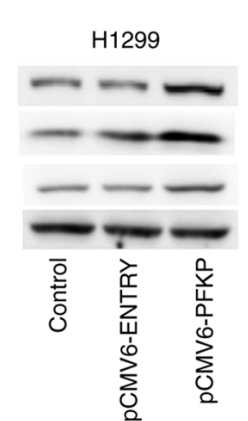
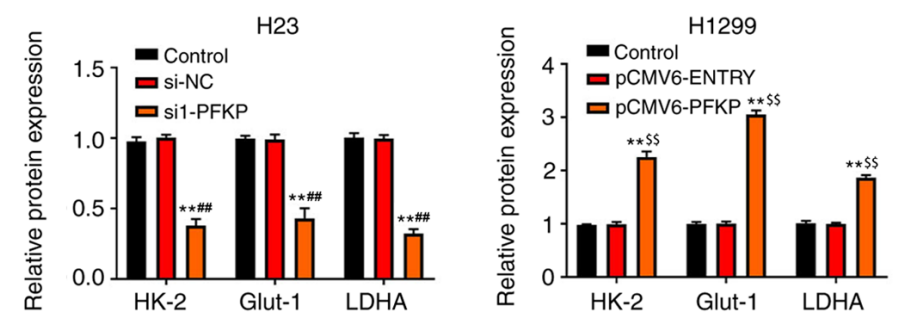

Figure 5. PFKP promotes glycolysis of NSCLC cells. (A) Relative glucose uptake. (B) Relative lactate production. (C) ATP/ADP ratio. (D) Expression levels of glycolysis-associated enzymes (HK-2, LDHA and Glut-1) were detected by western blot analysis. Data are presented as mean \pm SD from three independent experiments. ${ }^{* *} \mathrm{P}<0.01$ vs. control group; ${ }^{\# \#} \mathrm{P}<0.01$ vs. si-NC group; ${ }^{\mathrm{S}} \mathrm{P}<0.01$ vs. $\mathrm{pCMV6-ENTRY}$ group. PFKP, platelet isoform of phosphofructokinase; ATP, adenosine triphosphate; ADP, adenosine diphosphate; HK-2, hexokinase-2; LDHA, lactate dehydrogenase A; Glut-1, glucose transporter-1; si, small interfering RNA; NC, negative control.
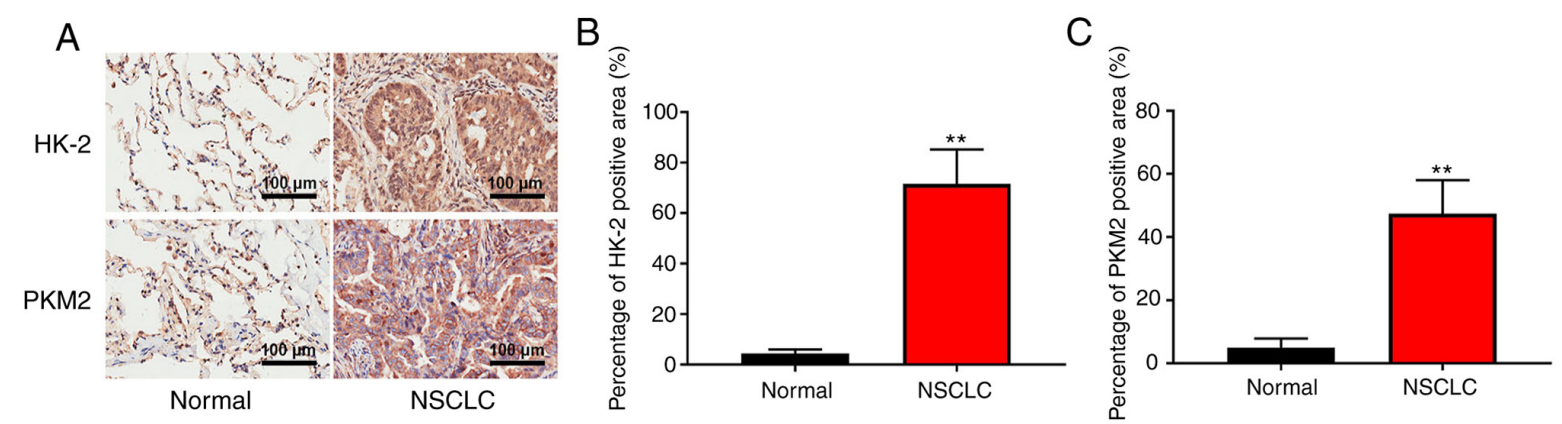

Figure 6. PFKP may increase the expression of HK-2 and PKM2, and regulate glycolysis in NSCLC. (A) Representative images of immunohistochemical staining of HK-2 and PKM2 in patient samples (NSCLC and corresponding adjacent tissues). Scale bar, $100 \mu \mathrm{m}$. Immunohistochemical analysis was used to detect (B) HK-2 and (C) PKM2 expression in NSCLC and corresponding adjacent tissues (normal) $(\mathrm{n}=84) .{ }^{* *} \mathrm{P}<0.01$ vs. normal tissues. PFKP, platelet isoform of phosphofructokinase; NSCLC, non-small cell lung cancer; HK-2, hexokinase-2; PKM2, pyruvate kinase M2.

To develop novel treatment strategies for cancer, accumulating evidence has demonstrated that biomarkers are associated with cancer progression $(28,29)$. Previously, it has been reported that the expression of PFKP is upregulated in most types of cancer and is associated with prognosis (30). In addition, it has been reported that the expression of PFKP in oral squamous cell carcinoma tissues is higher than that in adjacent noncancerous tissues, and that it is associated with differentiation and lymph node metastasis (31). Lee et al (30) suggested that PFKP expression in human glioblastoma (GBM) specimens may be upregulated compared with that in normal human brain tissue, and PFKP expression was shown to be increased in primary GBM cells. Consistent with these previous results, the present study demonstrated that PFKP expression was upregulated in NSCLC tissues and cells, and was associated with lymph node metastasis and histological grade. These findings suggested that PFKP may have an important role in the carcinogenesis of NSCLC. 
Phosphofructokinase-1 (PFK-1) is a key rate-limiting enzyme that has a critical role in the glycolytic pathway (32). PFK-1 has three isoforms: Liver isoform of phosphofructokinase (PFKL), muscle isoform of phosphofructokinase and PFKP (33). It has been reported that knockdown of PFK-1 expression may inhibit cancer cell proliferation and tumorigenicity (34). Yang et al (35) demonstrated that knockdown of PFKL inhibited cell growth and colony formation in H460 and H1299 cells, whereas overexpression of PFKL promoted cell growth and colony formation. Moreover, a study by Wang et al (21) indicated that knockdown of PFKP suppressed cell proliferation, promoted apoptosis and induced cell cycle arrest in kidney cancer cells. In the present study, overexpression of PFKP promoted cell proliferation, inhibited apoptosis, reduced the protein expression of caspase- 3 and increased the protein expression of Bcl-2 in H1299 cells, whereas knockdown of PFKP in H23 cells had the opposite effect. Taken together, these findings indicated that PFKP may promote cell proliferation and inhibit apoptosis of NSCLC cells.

Metastasis is the main cause of death in patients with cancer (36). EMT is reported to be an important cause of distal metastases of malignant tumors (37). Accumulating evidence has demonstrated that metastatic potential can be enhanced by activating EMT in numerous types of cancer, including HCC (38), BC (39) and cervical cancer (40). The present study revealed that overexpression of PFKP decreased the protein expression levels of E-cadherin, and increased the protein expression levels of $\mathrm{N}$-cadherin and vimentin, whereas knockdown of PFKP had the opposite effect. In addition, overexpression of PFKP increased the wound healing rate and number of invasive cells among H1299 cells, but knockdown of PFKP decreased the wound healing rate and number of invasive cells among H23 cells. These results suggested that PFKP may promote migration, invasion and EMT in NSCLC cells.

The Warburg effect serves a critical role in tumor development (41). Aerobic glycolysis is the main energy supply mode for tumor cells (42). During glycolysis, glucose is converted to pyruvate to accumulate lactate, which is accompanied by ATP production $(43,44)$. Moreover, a number of enzymes (including HK2, GLUT1, LDHA and PKM2) are involved in glycolysis $(45,46)$. Wang et al $(21)$ reported that knockdown of PFKP reduced glucose uptake and lactate production in kidney cancer cells. In the present study, the results revealed that overexpression of PFKP enhanced glucose uptake, lactate production and the ATP/ADP ratio, and increased the protein expression of HK-2, LDHA and Glut-1 in H1299 cells, whereas knockdown of PFKP reduced glucose uptake, lactate production and the ATP/ADP ratio, and decreased the protein expression of HK-2, LDHA and Glut-1 in H23 cells. Moreover, the present results showed that the expression levels of HK-2 and PKM2 in NSCLC tissues were higher than those in normal tissues. These results suggested that PFKP may promote the protein expression of HK-2, LDHA, Glut-1 and PKM2, and regulate glycolysis in NSCLC.

In conclusion, PFKP expression in NSCLC tissues and cells was upregulated, and was associated with lymph node metastasis and histological grade. Moreover, PFKP inhibited cell apoptosis, and promoted proliferation, migration, invasion and glycolysis in NSCLC cells. Although Kaplan-Meier survival analysis of TCGA data was performed in the present study, further studies, such as Kaplan-Meier survival analysis of clinical data, are required to investigate the prognostic value of PFKP. However, the present study provides a deeper understanding of NSCLC progression, which may provide a foundation for the treatment of NSCLC.

\section{Acknowledgements}

Not applicable.

\section{Funding}

No funding was received.

\section{Availability of data and materials}

The datasets used and/or analyzed during the current study are available from the corresponding author on reasonable request.

\section{Authors' contributions}

ZZ designed the study. FW and LL performed the research, analyzed the data and wrote the paper. All authors read and approved the final manuscript.

\section{Ethics approval and consent to participate}

The present study was approved by the Ethics Committee of Shandong Provincial Hospital (grant no. 2020-181). All patients provided written informed consent.

\section{Patient consent for publication}

Not applicable.

\section{Competing interests}

The authors declare that they have no competing interests.

\section{References}

1. Siegel RL, Miller KD and Jemal A: Cancer statistics, 2018. CA Cancer J Clin 68: 7-30, 2018.

2. Walker S: Updates in non-small cell lung cancer. Clin J Oncol Nurs 12: 587-596, 2008.

3. Shao Y, Liang B, Long L and Jiang SJ: Diagnostic MicroRNA biomarker discovery for non-small-cell lung cancer adenocarcinoma by integrative bioinformatics analysis. Biomed Res Int 2017: 2563085, 2017.

4. Wu C, Zhu W, Qian J, He S, Wu C, Chen Y and Shu Y: WT1 promotes invasion of NSCLC via suppression of CDH1. J Thorac Oncol 8: 1163-1169, 2013.

5. De Mukherjee K, Vats A, Ghosh D and Pillai SK: Data analysis and network study of non-small-cell lung cancer biomarkers. In: Advances in Computational Intelligence. Sahana $\mathrm{S}$ and Bhattacharjee V (eds). Springer, Singapore, pp265-272, 2020.

6. Liao Y, Cao L, Wang F and Pang R: miR-605-5p promotes invasion and proliferation by targeting TNFAIP3 in non-small-cell lung cancer. J Cell Biochem 121: 779-787, 2020.

7. Li Z, Li X, Wu S, Xue M and Chen W: Long non-coding RNA UCA1 promotes glycolysis by upregulating hexokinase 2 through the mTOR-STAT3/microRNA143 pathway. Cancer Sci 105: 951-955, 2014. 
8. Liu X, Wang X, Zhang J, Lam EK, Shin VY, Cheng AS, Yu J, Chan FK, Sung JJ and Jin HC: Warburg effect revisited: An epigenetic link between glycolysis and gastric carcinogenesis. Oncogene 29: 442-450, 2010

9. Meienhofer Mc, De Medicis E, Cognet M and Kahn A: Regulation of genes for glycolytic enzymes in cultured rat hepatoma cell lines. Eur J Biochem 169: 237-243, 1987.

10. Brand K: Aerobic glycolysis by proliferating Cells: Protection against oxidative stress at the expense of energy yield. J Bioenerg Biomembr 29: 355-364, 1997.

11. Gillies RJ, Robey I and Gatenby RA: Causes and consequences of increased glucose metabolism of cancers. J Nucl Med 49 (Suppl 2): 24S-42S, 2008

12. Lang N, Wang C,Zhao J, Shi F, Wu T and Cao H: Long non-coding RNA BCYRN1 promotes glycolysis and tumor progression by regulating the miR-149/PKM2 axis in non-small-cell lung cancer. Mol Med Rep 21: 1509-1516, 2020.

13. Gan J, Li S, Meng Y, Liao Y, Jiang M, Qi L, Li Y and Bai Y: The influence of photodynamic therapy on the Warburg effect in esophageal cancer cells. Lasers Med Sci 35: 1741-1750, 2020.

14. Li J, Cheng D, Zhu M, Yu H, Pan Z, Liu L, Geng Q, Pan H, Yan M and Yao M: OTUB2 stabilizes U2AF2 to promote the Warburg effect and tumorigenesis via the AKT/mTOR signaling pathway in non-small cell lung cancer. Theranostics 9: 179-195, 2019

15. Shi J, Wang H, Feng W, Huang S, An J, Qiu Y and Wu K MicroRNA-130a targeting hypoxia-inducible factor 1 alpha suppresses cell metastasis and Warburg effect of NSCLC cells under hypoxia. Life Sci 255: 117826, 2020.

16. Liu $\mathrm{T}$ and Yin H: PDK1 promotes tumor cell proliferation and migration by enhancing the Warburg effect in non-small cell lung cancer. Oncol Rep 37: 193-200, 2017.

17. Park YY, Kim SB, Han HD, Sohn BH, Kim JH, Liang J, Lu Y, Rodriguez-Aguayo C, Lopez-Berestein G, Mills GB, et al: Tat-activating regulatory DNA-binding protein regulates glycolysis in hepatocellular carcinoma by regulating the platelet isoform of phosphofructokinase through microRNA 520 . Hepatology 58: 182-191, 2013.

18. Zhang YM, Liu JK and Wong TY: The DNA excision repair system of the highly radioresistant bacterium Deinococcus radiodurans is facilitated by the pentose phosphate pathway. Mol Microbiol 48: 1317-1323, 2003

19. Chen Y, Xu Q, Ji D, Wei Y, Chen H, Li T, Wan B, Yuan L, Huang R and $\mathrm{Chen} \mathrm{G}$ : Inhibition of pentose phosphate pathway suppresses acute myelogenous leukemia. Tumour Biol 37: 6027-6034, 2016.

20. Kim NH, Cha YH, Lee J, Lee SH, Yang JH, Yun JS, Cho ES Zhang X, Nam M, Kim N, et al: Snail reprograms glucose metabolism by repressing phosphofructokinase PFKP allowing cancer cell survival under metabolic stress. Nat Commun 8: 14374, 2017.

21. Wang J, Zhang P, Zhong J, Tan M, Ge J, Tao L, Li Y, Zhu Y, Wu L, Qiu J and Tong X: The platelet isoform of phosphofructokinase contributes to metabolic reprogramming and maintains cell proliferation in clear cell renal cell carcinoma. Oncotarget 7: 27142-27157, 2016

22. Liu J, Lichtenberg T, Hoadley KA, Poisson LM, Lazar AJ, Cherniack AD, Kovatich AJ, Benz CC, Levine DA, Lee AV, et al: An integrated TCGA pan-cancer clinical data resource to drive high-quality survival outcome analytics. Cell 173: 400-416.e11, 2018

23. Love MI, Huber W and Anders S: Moderated estimation of fold change and dispersion for RNA-seq data with DESeq2. Genome Biol 15: 550, 2014.

24. Therneau T M and Grambsch P M (eds): Modeling Survival Data: Extending the Cox Model. Springer, New York, NY, 2000.

25. Livak KJ and Schmittgen TD: Analysis of relative gene expression data using real-time quantitative PCR and the 2(-Delta Delta C(T)) method. Methods 25: 402-408, 2001.

26. Reck M,Heigener DF, Mok T, Soria JC and Rabe KF: Management of non-small-cell lung cancer: Recent developments. Lancet 382: 709-719, 2013

27. Mor I, Cheung EC and Vousden KH: Control of glycolysis through regulation of PFK1: Old friends and recent additions. Cold Spring Harb Symp Quant Biol 76: 211-216, 2011

28. Wang C, Chen S, Wang Y, Liu X, Hu F, Sun J and Yuan H: Lipase-triggered water-responsive 'Pandora's Box' for cancer therapy: Toward induced neighboring effect and enhanced drug penetration. Adv Mater 30: e1706407, 2018.
29. Tang D, Zhao X, Zhang L, Wang Z and Wang C: Identification of hub genes to regulate breast cancer metastasis to brain by bioinformatics analyses. J Cell Biochem 120: 9522-9531, 2019.

30. Lee JH, Liu R, Li J, Zhang C, Wang Y, Cai Q, Qian X, Xia Y, Zheng Y, Piao Y, et al: Stabilization of phosphofructokinase 1 platelet isoform by AKT promotes tumorigenesis. Nat Commun 8: 949, 2017.

31. Chen G, Liu H, Zhang Y, Liang J, Zhu Y, Zhang M, Yu D, Wang $\mathrm{C}$ and Hou J: Silencing PFKP inhibits starvation-induced autophagy, glycolysis, and epithelial mesenchymal transition in oral squamous cell carcinoma. Exp Cell Res 370: 46-57, 2018.

32. Wegener $\mathrm{G}$ and Krause U: Different modes of activating phosphofructokinase, a key regulatory enzyme of glycolysis, in working vertebrate muscle. Biochem Soc Trans 30: 264-270, 2002.

33. Moon JS, Kim HE, Koh E, Park SH, Jin WJ, Park BW, Park SW and Kim KS: Krüppel-like factor 4 (KLF4) activates the transcription of the gene for the platelet isoform of phosphofructokinase (PFKP) in breast cancer. J Biol Chem 286: 23808-23816, 2011.

34. Yi W, Clark PM, Mason DE, Keenan MC, Hill C, Goddard WA III, Peters EC, Driggers EM and Hsieh-Wilson LC: Phosphofructokinase 1 glycosylation regulates cell growth and metabolism. Science 337: 975-980, 2012.

35. Yang J, Li J, Le Y, Zhou C, Zhang S and Gong Z: PFKL/miR-128 axis regulates glycolysis by inhibiting AKT phosphorylation and predicts poor survival in lung cancer. Am J Cancer Res 6: 473-485, 2016

36. Tam WL and Weinberg RA: The epigenetics of epithelial-mesenchymal plasticity in cancer. Nat Med 19: 1438-1449, 2013.

37. Zhou P, Li B, Liu F, Zhang M, Wang Q, Liu Y, Yao Y and Li D: The epithelial to mesenchymal transition (EMT) and cancer stem cells: Implication for treatment resistance in pancreatic cancer. Mol Cancer 16: 52, 2017.

38. Ma P, Tang WG, Hu JW, Hao Y, Xiong LK, Wang M, Liu H, Bo WH and Yu KH: HSP4 triggers epithelial-mesenchymal transition and promotes motility capacities of hepatocellular carcinoma cells via activating AKT. Liver Int 40: 1211-1223, 2020.

39. Xiao Y, Xie Q, Qin Q, Liang Y, Lin H and Zeng D: Upregulation of SOX11 enhances tamoxifen resistance and promotes epithelial-to-mesenchymal transition via slug in MCF-7 breast cancer cells. J Cell Physiol 235: 7295-7308, 2020.

40. Yang L, Yu Y, Xiong Z, Chen H, Tan B and Hu H: Downregulation of SEMA4C inhibit epithelial-mesenchymal transition (EMT) and the invasion and metastasis of cervical cancer cells via inhibiting transforming growth factor-beta 1 (TGF- $\beta 1$ )-induced hela cells p38 mitogen-activated protein kinase (MAPK) activation. Med Sci Monit 26: e918123, 2020.

41. Yang W, Zheng Y, Xia Y, Ji H, Chen X, Guo F, Lyssiotis CA, Aldape K, Cantley LC, Lu Z, et al: ERK1/2-dependent phosphorylation and nuclear translocation of PKM2 promotes the Warburg effect. Nat Cell Biol 14: 1295-1304, 2012

42. Liu L, Wang Y, Bai R, Yang K and Tian Z: MiR-186 inhibited aerobic glycolysis in gastric cancer via HIF-1 $\alpha$ regulation. Oncogenesis 6: e318, 2017.

43. Akram M: Mini-review on glycolysis and cancer. J Cancer Educ 28: 454-457, 2013

44. Ganapathy-Kanniappan S: Molecular intricacies of aerobic glycolysis in cancer: Current insights into the classic metabolic phenotype. Crit Rev Biochem Mol Biol 53: 667-682, 2018

45. Wan W, Peng K, Li M, Qin L, Tong Z, Yan J, Shen B and Yu C: Histone demethylase JMJD1A promotes urinary bladder cancer progression by enhancing glycolysis through coactivation of hypoxia inducible factor $1 \alpha$. Oncogene 36 : 3868-3877, 2017.

46. Xu H, Zeng Y, Liu L, Gao Q, Jin S, Lan Q, Lai W, Luo X, Wu H, Huang Y and Chu Z: PRL-3 improves colorectal cancer cell proliferation and invasion through IL-8 mediated glycolysis metabolism. Int J Oncol 51: 1271-1279, 2017.

This work is licensed under a Creative Commons Attribution-NonCommercial-NoDerivatives 4.0 International (CC BY-NC-ND 4.0) License. 